PostGRAD. MED. J. (1965), 41, 325

\title{
ELECTRODIAGNOSIS OF MYOPATHIC DISORDERS
}

\author{
D. A. H. YateS, M.D., M.R.C.P., D.Phys.Med. \\ Chief Assistant, Department of Physical Medicine, St. Thomas's Hospital, London.
}

ELECTRODIAGNOSTIC examination (EMG) can be of considerable help in the diagnosis of various muscle disorders. However, the electrical abnormalities of diseased muscle are rarely pathognomonic of one particular condition and, as with other specialised techniques, the results are of most value when correlated with the results of other investigations.

The examiner should fully appraise the history, the clinical state of the patient and other available data in order to decide which muscles to study and which procedures will produce the most information with the least disturbance to the patient.

\section{Equipment}

A standard electromyograph contains a muscle stimulator unit, a nerve stimulator and a high gain multi-stage amplifier with a high discrimination ratio and a high input impedance. The muscle stimulator unit delivers rectangular pulses of variable amplitude and preset duration at regular intervals. The nerve stimulator unit is isolated from earth to minimize the shock artefact in the recording, and is designed to trigger the sweep of the display oscilloscope, thus providing for the accurate measurement of the latency of the muscle response.

For recording electrical potentials in muscle, unipolar concentric electrodes are generally used. In structure these consist of a hypodermic needle down the centre of which is threaded a fine insulated wire, the tip of this wire being the active recording surface. Following amplification the signals are displayed on a cathode-ray oscilloscope with a time scale providing 10 and 1 millisecond divisions and an oscilloscope camera produces permanent records for more detailed analysis. The amplified signals are also fed into a loud speaker for auditory monitoring.

\section{Electrodiagnostic Procedures}

1. Nerve Stimulation.

- Threshold of Excitation.

-Anomalous Innervation.

-Response to Repetitive Stimulation.

2. Direct Stimulation of Muscle.

-Intensity-duration Relationships.

-Briskness of Response.

3. Electromyographic Exploration of Muscle. $\rightarrow$ Spontaneous Activity at Rest.

- Volitional Activity.

- Mean Potential Duration Estimation -Motor Nerve Conduction Studies. -Multi-electrode Studies.

4. Pharmacological Tests.

These procedures, when used in combination, will indicate whether the lesion affects primarily the muscle fibres as in the myopathies, the neuromuscular junction as in myasthenia and myotonia, the lower motor neurone as in the neuropathies, or the spinal cord as in the myelopathies.

\section{Nerve Stimulation}

The response to the stimulation of peripheral nerves often gives useful information, and should be performed routinely. If there is a lesion of one peripheral nerve its threshold to stimulation may be found to be considerably higher than that of other nerves, while in polyneuritis the thresholds of all peripheral nerves may be raised. The pattern of muscle response may reveal anomalous innervation or indicate a primary agenesis of an individual muscle. Repetitive stimulation at different rates up to that producing a sustained tetanus is used in investigating the myasthenic disorders and the effects of various pharmacological agents can be observed.

\section{Direct Stimulation of Muscle}

Skeletal muscle which has been partially or totally deprived of its nerve supply shows an 


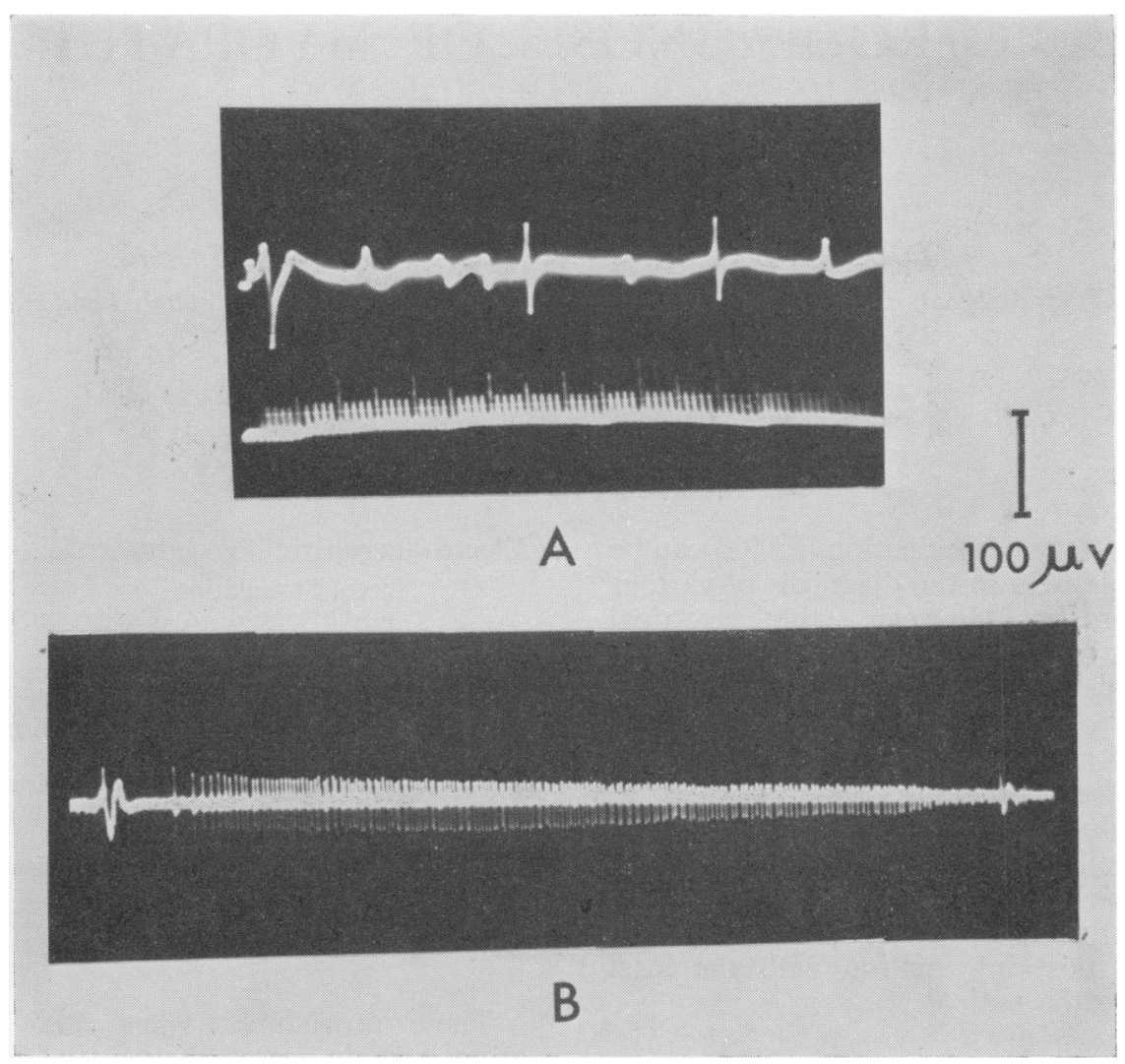

FIG. 1.-A. Fibrillation and positive potentials.

B. High-frequency discharge.

Time scale in 1 and $10 \mathrm{msec}$. divisions.

increased threshold to short-duration stimuli. The plotting of intensity-duration curves can be time-consuming and Bauwens (1943) has advocated an abbreviated form of this investigation to enable more muscles to be examined. He has shown in normal muscle, using currentstabilised pulses, that the threshold for a 1 msec. duration stimulus does not exceed twice the threshold to stimuli of 10 msecs. and 100 msecs. duration, the latter being the equivalent of the rheobase. In partially denervated muscle the 1 msec. threshold exceeds twice the rheobase, while in totally denervated muscle it may be so high that a response cannot be obtained. When a muscle is severely fibrosed or agenetic no response can be obtained even to the long duration stimulus.

\section{Electromyographic Exploration of Muscle}

The insertion of a needle electrode into a denervated portion of muscle at rest may evoke short duration diphasic fibrillation potentials which can be recognised by their characteristic regular clicking sound on the loud speaker. Another characteristic discharge of denervated muscle is the "positive'? potential which is a monophasic spike of longer duration and is heard as a series. of regular, dull thuds (Fig. 1a). In the myotonic disorders the neuromuscular junction appears to be hyper-excitable. Any mechanicab stimulation of the muscle will evoke a train of high frequency discharges which produce a characteristic "dive-bomber" sound. (Fig. 1b)

The functional unit in reflex or voluntary activity is the motor unit which comprises arv anterior horn cell, its neurone and branches and the group of muscle fibres which they innero vate. Conduction along the fine intramuscular branches of the lower motor neurone occure్ so rapidly that all the muscle fibres in a motote unit are activated almost simultaneously. Theo mean number of muscle fibres per motor uni $\overline{0}$ varies considerably from muscle to muscle 


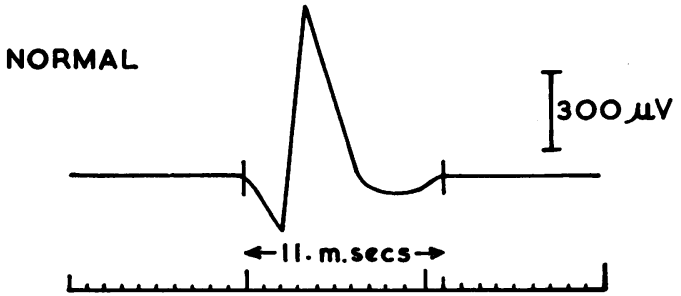

MYOPATHY

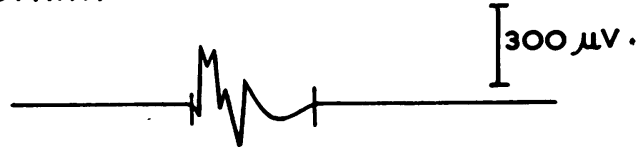

7. m.secs

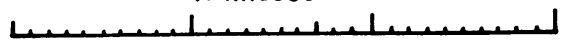

FIG. 2.-Diagrammatic representation of individual muscle potentials recorded at minimal volition to show technique of duration measurement. Time scale 1 and $10 \mathrm{msec}$.

those of gastrocnemius average about 1600 muscle fibres while those of the ocular muscles contain less than 10 fibres. In the limb muscles these fibres are grouped in 50 to 100 sub-units which lie interspersed with sub-units innervated by other anterior horn cells.

The active core of a concentric needle electrode will record spike potentials arising within a distance of $0.5 \mathrm{~mm}$. and within this area will lie some 10 to 20 different sub-units. The electrical potentials produced by these sub-units, somewhat confusingly termed motor unit potentials, summate during increasing volitional effort as increasing numbers are activated to a complete interference pattern. The electrical activity produced by increasing volitional effort indicates whether normal and full recruitment of motor units occurs. At minimal volitional effort it is possible to study the duration, configuration and amplitude of individual motor unit potentials, particularly if photographic records are made. A useful diagnostic parameter is to measure the durations of at least twenty different potentials and obtain the mean of these durations. (Fig. 2). The normal range of mean potential duration (MPD) can be obtained for individual muscles by studying control subjects of different ages, and Fig. 3 shows that the MPD increases with age. The measurement is affected by other factors, particularly by the electrode characteristics, so that independent control figures must be established by each

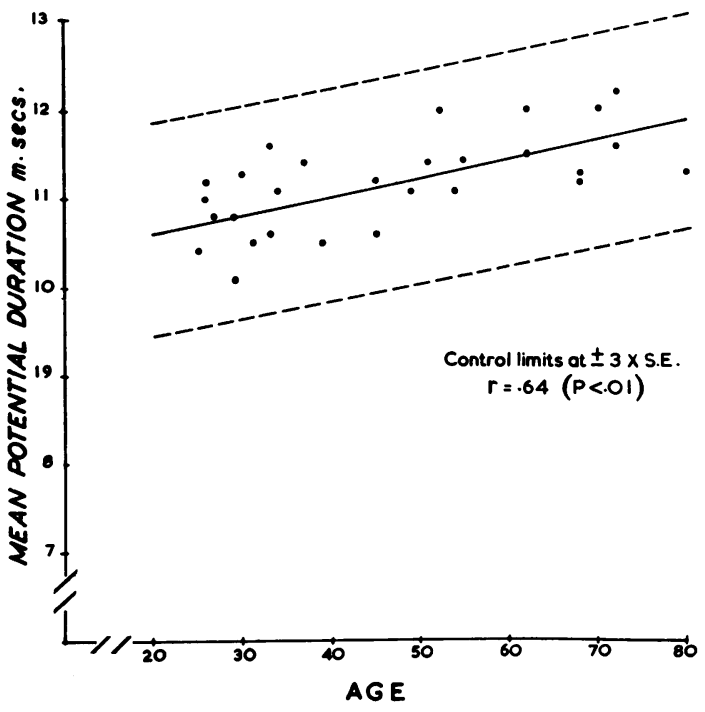

FIG. 3.-Relation between age (in years) and mean potential duration (MPD) in deltoid muscle in 28 control subjects. Equation of regression line is : $\mathrm{MPD}=0.020 \times$ age +10.21 .

Broken lines are 3 standard deviations $(3 \times 0.40$ msec. $=1.20 \mathrm{msec}$.) either side of the regression line; these are taken as the normal limits.

laboratory (Buchthal, Gold and Rosenfalck, 1954).

Since the velocity of conduction along a nerve is proportional to its diameter, a localised constriction of a peripheral nerve or a diffuse thinning of its fibres will reduce the rate of conduction. The rate of motor nerve conduction can be calculated from the latency of the muscle response when its nerve is stimulated at different levels. In a normal peripheral nerve the fibres have conduction rates of the same order and evoke a synchronised response in the muscle. However, if for any reason the conduction rate in some of the nerve fibres is reduced, this will produce a temporal dispersion of the nerve impulses which will be recorded as a scatter of the response in the muscle.

By using a multi-electrode probe, Buchthal, Erminio and Rosenfalck (1959) were able to measure the spatial distribution of muscle fibres innervated by a single anterior horn cell, the motor unit territory.

\section{Electrodiagnostic Changes in Muscle Fibre Lesions}

In primary muscle-fibre disease the peripheral nerves and muscles usually respond normally to electrical stimulation unless gross 


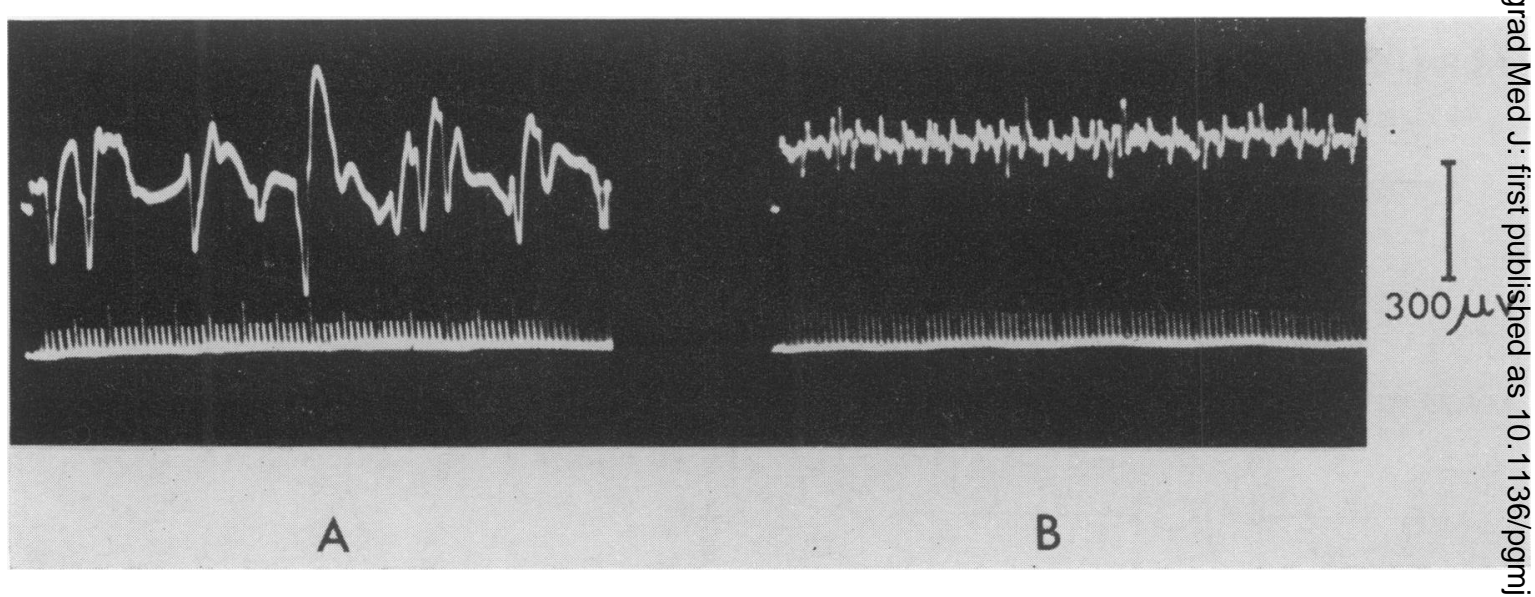

FIG. 4.-Full volitional activity patterns.

A. Normal muscle.

B. Myopathic muscle.

Time scale in 1 and $10 \mathrm{msec}$. divisions.

fibrosis and contracture have occurred. Occasionally fibrillation potentials, the hallmark of denervation, may be detected in polymyositis, and rarely in the muscular dystrophies, and this is probably due to some secondary involvement of terminal nerve fibres. Profuse high-frequency discharges are detected in dystrophia myotonica and myotonia congenita but may also be found to a lesser extent in polymyositis, hyperkalaemic periodic paralysis and myxoedema.

The various myopathic disease processes pick off individual muscle fibres from the subunits which consequently produce action potentials that are shorter in duration, and smaller in amplitude, a greater proportion than normal are polyphasic (Fig. 4), but these potentials can still summate to a semblance of an interference pattern until the late stages of the disease cause gross muscle destruction. These changes increase the high-frequency components in the electrical activity produced on volition which can be detected as a "crackliness" on the loud speaker or by electronic frequency analysis. However, these changes are frequently only detectable in some areas of a myopathic muscle while other areas of the same muscle seem normal. A quantitative and objective estimate of these changes is thus desirable but this is difficult to obtain from frequency analysis. The MPD has been shown to be shortened in myopathic disorders (Kugelberg, 1949), in polymyositis (Buchthal and Pinelli, 1953) and in the myopathies complicating thyrotoxicosis, Cushing's Syndrome and corticosteroid therapy (Yates, 1963). This method produces a quantitative result, and Fig. 5 shows the results obtained in sixteen cases of myopathy due to various causes. The results of serial examinations can be compared as in $\overrightarrow{8}$ reversible myopathies complicating endocriner disorders (Fig. 6). where the MPD tends return to normal with recovery.

Similar electromyographic changes occur in the myopathies occasionally encountered in other diseases, such as carcinoma, dissemin ated lupus erythematosis, polyarteritis nodosä and rheumatoid arthritis but the EMG provides no particular differentiating signs.

Buchthal, Rosenfalck and Erminio (1960 have shown a reduction of motor-unit terri tory in myopathic disorders but the techniques requires special equipment and is too time? consuming for routine use. Other methods are available by which the electrical changes occurring in myopathy can be quantitated. If the complex electrical activity produced on volition is passed through an integrating cir을. cuit following amplification the mean voltage can be measured. Lenman (1959) has related the integrated electrical activity produced by $\mathrm{a}$ muscle to the mechanical tension achieved and has shown that myopathic muscle produces relatively less contractile force than normab muscle at a similar level of electrical activity Using a special tracing device Willison (1964) has shown that in a myopathic muscle the electrical activity pattern shows a higher spike frequency and sometimes a lower mean spik@ 


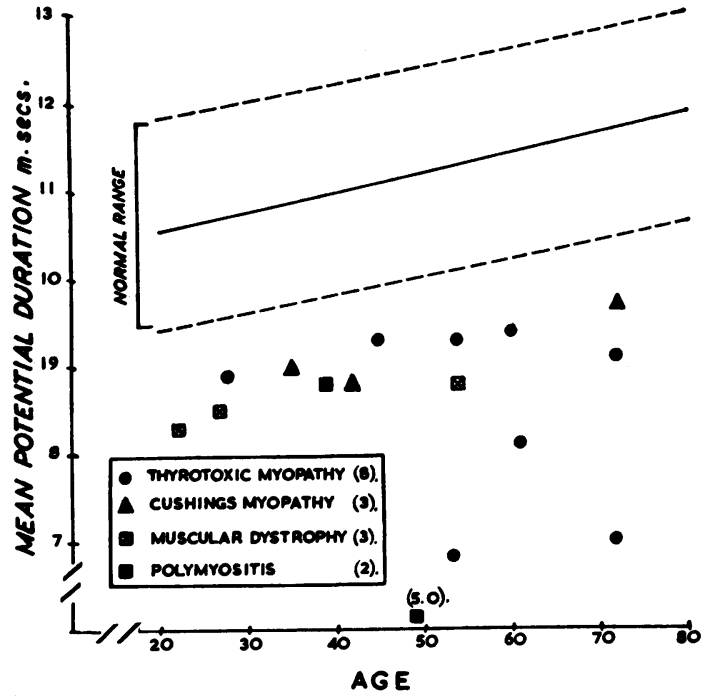

Fig. 5.- Mean potential duration in deltoid muscle in sixteen cases of myopathy.

amplitude than in a normal muscle operating against the same resistance. Further automation of both these techniques is required before their application to routine diagnostic work can be assessed and their reliability in detecting minor degrees of myopathic involvement can be estimated. At present the estimation of MPD seems to be the most reliable method that can be performed on standard electromyographs.

In the later stages of dystrophia myotonica muscle fibre degeneration occurs and the EMG changes of myopathy develop in addition to the myotonia. Sarcoidosis may be complicated by a myopathy (Crompton and MacDermott, 1961), but in addition abnormal nerve conduction studies and signs of denervation in peripheral muscle may indicate a peripheral neuritis.

Muscle wasting occurs as a result of disuse particularly in relation to painful joints. The only EMG abnormality that can be detected in atrophic muscle is an inability to produce a complete interference pattern on volition, while the individual motor unit potentials have a normal duration.

\section{Myasthenic Syndromes}

Various electro-pharmacological tests have been introduced to investigate these conditions but the techniques and their interpretation are complicated. For detailed information the reader is referred to a recent review by Simpson

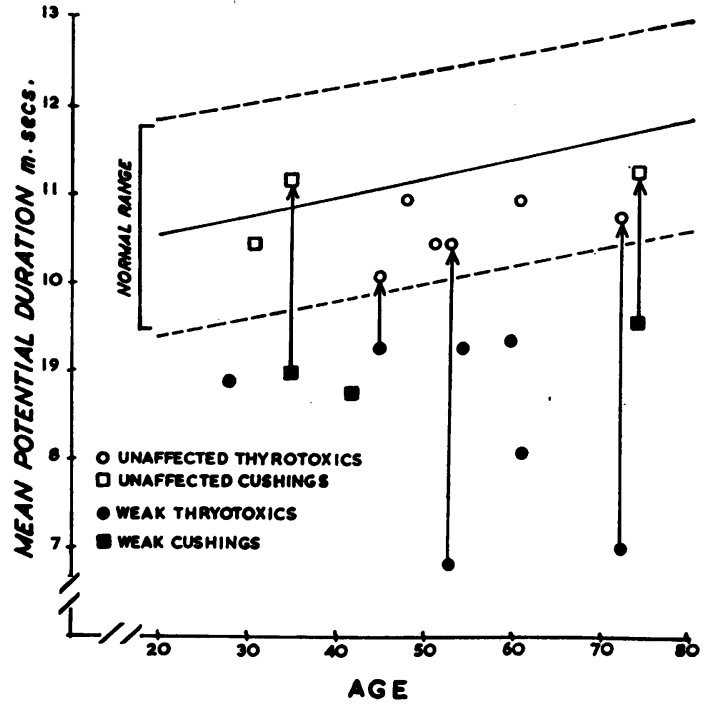

FIG. 6.-Mean potential duration in deltoid muscle in 10 cases of thyrotoxicosis and four cases of Cushing's syndrome showing correlation between clinical weakness and shortening of mean potential duration. Upward pointing arrows indicate return of normality following successful treatment.

(1964) which gives a full bibliography and only a summary will be given here.

\section{Myasthenia Gravis}

EMG recording shows that the loss of power which occurs when the motor nerve to an affected muscle is repetitively and supramaximally stimulated is accompanied by a decrement in the size of the evoked muscle action potentials. This phenomenon can usually be prevented or reversed by the intravenous administration of edrophonium hydrochloride ('Tensilon'), a short-acting anticholinesterase substance. The muscles of a myasthenic subject are more resistant to the neuromuscular blocking action of decamethonium iodide (C 10) than normal muscles. When a neuromuscular block is produced by this agent its action can be reversed by edrophonium in myasthenic muscle but not in normal muscle. (Churchill-Davidson and Richardson, 1952). This test should only be performed with the full measures for assisted respiration immediately available. When the disease is long-standing permanent myopathic changes develop in weakened muscles.

\section{Carcinomatous Myasthenia}

Anderson, Churchill-Davidson and Richardson, (1952) reported a myasthenic syndrome 
associated with a bronchial carcinoma presenting as an undue sensitivity to succinylcholine. Lambert, Eaton and Rooke, (1956) described six cases with pulmonary neoplasm and associated myasthenia. On repetitive nerve stimulation a marked increment (up to tenfold) was observed in the evoked muscle response. Tetanic facilitation, as this phenomenon is called, is not peculiar to this condition but when it is observed thorough investigation including tomography of both lung fields is indicated.

Fatigue phenomena which can be reversed by edrophonium and other anticholinesterases are also sometimes encountered in a variety of neurological disorders including polymyositis, muscular dystrophy and motorneurone disease.

\section{Metabolic Diseases of Muscle}

Hypokalaemic periodic paralysis is a familial condition, characterised by attacks of weakness lasting several hours, associated with a low plasma potassium and which are often precipitated by heavy carbohydrate ingestion. During an attack the threshold of peripheral nerves and muscles to stimulation is markedly raised and no electrical activity is recorded by intramuscular electrodes. Eventually the muscles may show the EMG changes of myopathy in between attacks.

Hyperkalaemic periodic paralysis is also a familial condition but attacks are associated with a raised plasma potassium. During an attack the nerves and muscles are relatively inexcitable but intramuscular electrodes record fibrillation potentials and high frequency discharges, and this feature helps to distinguish this disease from hypokalaemic paralysis (Morrison, 1960). Here again, the muscles may eventually develop myopathic changes.

Weakness is a common complaint of patients with myxoedema. Astrom, Kugelberg and Muller (1961) claimed to have detected clinical and EMG evidence of myopathy in 5 out of 7 cases. However, Herring and Yates (1965) in a detailed study of ten cases with typical slow reflexes, found no clinical, electrodiagnostic or biochemical evidence of a true myopathy, and muscle biopsy showed normal histology apart from some infiltration of the muscle fibres with mucoid material in two cases.

In many of the myopathic disorders corticosteroid therapy has a beneficial effect. However, it has been shown that, even in conservative dosage, this therapy may produce the EMG changes of myopathy in previous normal muscles (Yates, 1936). To avoid con fusion an EMG examination must be pe formed before treatment is commenced to provide baseline data with which subsequent examinations can be compared.

It is important to avoid biopsy of a muscte which has recently been studied by EM because the insertion of needle electrodes causes a traumatic myositis which obscures the histological picture for some time. (Woolf 1962).

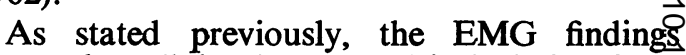
cannot be reliably interpreted in isolation fromb other data. The results should be correlatee with the clinical features, with the biops. histology and with the results of biochemica. tests which should preferably include the serunf aldolase and creatine phosphokinase levels: Nevertheless, in a recent study of polymyalgi® in which these factors were correlated. Chalmers, Alexander and Duthie (1964) cons cluded that EMG was the most useful single investigation in the diagnosis of myositis.

I am grateful to the Editor, British Medical Association Special Journals for permission $\delta \mathrm{tg}$ reproduce Figs. 2, 3, 5 and 6 from the articlesin which they originally appeared.

\section{REFERENCES}

Anderson, H. J., Churchill-Davidson, H. C., an Richardson, A. T. (1953): Bronchial Neoplasm with Myasthenia, Lancet, ii, 1291.

Astrom, K. E., KugelberG, E., and Muller, R (1961): Hypothyroid Myopathy, Arch. Neurol $5,472$.

BAUWENS, P. (1943): Electrodiagnostic Interpretations in Nerve Lesions, Proc. roy. Soc. Med., 3해 25.

Buchthal, F., and Pinelli, P. (1953): Muscle Actiong Potentials in Polymyositis, Neurology (Minneap 3, 424.

Buchthal, F., Gold, C., and Rosenfalck, P. (1954) Action Potential Parameters in NormalMuscle and their Dependence on Physical Variables Acta. physiol. scand., 32, 200.

Buchthal, F., ERMinio, F., and Rosenfalck, 帝 (1959): Motor Unit Territory in Different Muscleș Acta. physiol. scand., 45, 72.

Buchthal, F., Rosenfalck, P., and ERminio, $\mathfrak{H}_{0}$ (1960): Motor Unit Territory and Fibre Densit in Myopathies, Neurology (Minneap.), 10, 698.

Chalmers, T. M., Alexander, W. R. M., and Duthie, J. J. R. (1964): Polymyalgia: Problem of Differential Diagnosis, Ann. rheum. Dis., 28 123.

Churchill-Davidson, H. C., and Richardson, A. $\stackrel{\mathscr{T}}{\mathbf{T}}$ (1952): The Action of Decamethonium Iodide (C10) in Myasthenia Gravis, J. Neurol. Neurosur\$ Psychiat., 15, 129. 
Crompton, M. R., and MacDermott, V. (1961): Sarcoidosis Associated with Progressive Muscular Wasting and Weakness, Brain, 84, 62.

HeRring, A. B., and YATES, D. A. H. (1965): Muscular Changes in Myxoedema (in preparation). KugelberG, E. (1947): Electromyogram in Muscular Disorders, J. Neurol. Psychiat., 10, 122.

LAmbert, E. H., EATON, L. M., and RoOKe, E. D.

(1956): Defect of Neuromuscular Conduction Associated with Malignant Neoplasm, Amer. J. Physiol., 187, 612.

LENMAN, J. A. R. (1959): Quantitative Electromyographic Changes Associated with Muscular Weakness, J. Neurol. Neurosurg. Psychiat., 22, 306.

MORRISON, J. B. (1960): Electromyographic Changes in Hyperkalaemic Familial Periodic Paralysis, Ann. Phys. Med., 5, 153.
Simpson, J. A. (1964): Myasthenia Gravis and Myasthenic Syndromes in "Disorders of Voluntary Muscle", p. 336, ed. J. N. Walton, London: J. \& A. Churchill.

Willison, R. G. (1964): Analysis of Electrical Activity in Healthy and Dystrophic Muscle in Man, J. Neurol. Neurosurg. Psychiat., 27, 386.

Woolf, A. L. (1962): "Modern Trends in Neurology", 3rd series, p. 11, ed. D. Williams, London: Butterworths.

YATES, D. A. H. (1963a): The Estimation of Mean Potential Duration in Endocrine Myopathy, $J$. Neurol. Neurosurg. Psychiat., 26, 458.

YATES, D. A. H., (1936b): Muscular Changes in Rheumatoid Arthritis, Ann. rheum. Dis., 22, 342. 